\title{
3 Research Square \\ LPA3 Is a Precise Therapeutic Target And Potential Biomarker For Ovarian Cancer
}

\section{Pengfei Zhao}

Inner Mongolia University

\section{Qingru Yun}

Inner Mongolia University

\section{Aodungerile Li}

Inner Mongolia University

\section{Rong Li}

Inner Mongolia University

\section{Yali Yan}

Inner Mongolia University

\section{Yuewu Wang}

Inner Mongolia University

\section{Hongju Sun}

Inner Mongolia University

\section{Alatangaole Damirin ( $\nabla$ bigaole@imu.edu.cn )}

Inner Mongolia University https://orcid.org/0000-0001-6142-282X

\section{Research Article}

Keywords: LPA3, ovarian cancer, xenografted mice model, bioinformatics, signaling mechanism

Posted Date: September 20th, 2021

DOl: https://doi.org/10.21203/rs.3.rs-875460/v1

License: (c) (1) This work is licensed under a Creative Commons Attribution 4.0 International License.

Read Full License

Version of Record: A version of this preprint was published at Medical Oncology on January 4th, 2022. See the published version at https://doi.org/10.1007/s12032-021-01616-5. 


\section{Abstract}

Current studies have demonstrated that significant increased LPA levels to be observed in ascites in patients with ovarian cancer. Although several studies have shown that Lysophosphatidic acid (LPA) related to the progression of ovarian cancer, which LPA receptors (LPARs) and G coupled protein subtypes mediated in LPA actions have not been clearly elucidated. This study aimed to clarify the roles of LPA and it's subtype-specific LPARs mediating mechanisms in ovarian cancer by integrated using bioinformatic analysis and biological experimental approaches. The big data analysis shown that LPA3 was the only differentially expressed LPA receptor among the six LPARs in ovarian cancer and further verified in immunohistochemistry of tissue microarrays. Also found that LPA3 was also highly expressed in ovarian cancer tissue and ovarian cancer cells. Importantly, LPA significantly promoted the proliferation and migration of LPA3-overexpressing ovarian cancer cells, while the LPA-induced actions blocked by Ki16425, a LPAR1/3 antagonist treated, and LPA3-shRNA transfected. In vivo study indicated that the LPA3-overexpressing cell derived tumors metastasis, tumors volume and tumors mass were apparently increased in xenografted nude mice. In addition, we also observed that LPA3 was differential high-expression in ovarian cancer tissue of the patients. Our studies further confirmed the LPA3/Gi/MAPKs/NF-KB signals were involved in LPA-induced oncogenic actions in ovarian cancer cells. Our findings indicated that the LPA3 might be a novel precise therapeutic target and potential biomarker for ovarian cancer.

\section{Introduction}

Lipid signaling molecule LPA regulates multiple cellular functions, such as cell migration, proliferation and differentiation, as the ligand activates at least six known G protein-coupled LPA receptors (LPA1-6) involved in a variety of physiological and pharmacological processes[1]. Many studies have been reported that LPA accelerates occurrence and development of various cancers, including ovarian cancer, bladder cancer, brain cancer, stomach cancer, kidney cancer, and lung cancer[1-3]. According to the multiple studies reported, it was believed that the role and expression activity of LPA receptor subtypes in different types of tumors shown an obvious tissue specificity. Several research groups including our group reported that LPA1 closely related to lysophosphatidic acid-induced lung cancer, ovarian cancer and pancreatic cancer progression[4-6]. LPA2 and LPA3 was involved in the regulation of chemoresistance in cancer cells treated with $\operatorname{CDDP}[7,8]$. LPA was shown to be a biomarker for other gynecological cancers[9], as well as for gastric cancer and lung cancer[5, 10, 11]. LPARs, as small molecule targets shown to reflect a potential value in the drugs development of cancer therapy.

Ovarian cancer is a common gynecological malignancy with a high mortality rate[12]. Xu et al. firstly reported that LPA might be a potential biomarker for ovarian cancer due to high pathologic concentrations of plasma LPA in ovarian cancer patients[9]. The role of LPARs in ovarian cancer has attracted a lot of attention, such as, it was reported that LPA-induced metabolic adaptations and tumor aggressiveness mediated by LPA2 in ovarian cancer cells[13]. Subsequently, LPA-induced migration of ovarian cancer cells requires activation of ERM proteins via LPA1 and LPA2[14]. In addition, MicroRNA- 
15b targeting for LPAR3 inhibited the PI3K/Akt pathway to alleviate ovarian cancer[15]. The role of LPARs in ovarian cancer has caused widespread concern, but what remains unclear in the current studies is which LPA receptor subtype plays the most critical role in LPA-induced ovarian cancer progression. Therefore, our study will use big data analysis combined with biological methods to clarify the importance of LPA receptor subtypes in the development of ovarian cancer and trying to provide a clear answer to ambiguity in this research field.

\section{Material And Methods}

\section{Materials}

The A2780 (\#CL-0013) and SKOV3 (\#CL-0215) ovarian cancer cell lines were obtained from Procell Life Science \& Technology Co., Ltd. (Wuhan, China). HO-8910 was from our laboratory. A lentiviral vector was used to overexpress LPA3 in A2780, HO-8910 and SKOV3 cell lines. The reagents that contain LPA (C18:1, \#857230P), epidermal growth factor (EGF, \#SRP3027) and pertussis toxin (PTX, \#P7208) were purchased from Sigma-Aldrich. Other reagents were obtained from Selleck Chemicals, such as PD98059 (\#S1177), SP600125 (\#S1460), BAY11-7082 (\#S2913), AG1478 (\#S2728) and SB203580 (\#S1076). The anti-pEGFR (\#3777), anti-EGFR (\#2085), anti-p-ERK1/2 (\#9101), anti-ERK1/2 (\#4695), anti-p-JNK (\#4671), antiJNK (\#9252), anti-p38 (\#8690), anti-p-p38 (\#4511), anti-Akt (\#4691), anti-p-Akt (\#4060), anti- NF-kB (\#8242), anti-p-NF-KB (\#3033) and anti-a-tubulin (\#3873) were obtained from Cell Signaling Technology.

\section{Bioinformatics analysis}

The gene expression data of normal ovarian tissue were extracted from Genotype-Tissue Expression (GTEx) database. The gene expression data of ovarian cancer tissue were obtained from The Cancer Genome Atlas (TCGA) database. Then differential gene expression analysis was performed by $\mathrm{R}$ Language in ovarian cancer samples and normal ovarian samples.

\section{Cell viability assay}

When cells were attached to bottom of 96-well plates, changed to starvation medium for overnight. The cells were stimulated with EGF $(20 \mathrm{ng} / \mathrm{ml})$, PD98059 $(5 \mu \mathrm{M})$, LPA $(1 \mu \mathrm{M}), \mathrm{Ki} 16425(1 \mu \mathrm{M})$, SB203580 $(1$ $\mu \mathrm{M})$, PTX $(100 \mathrm{ng} / \mathrm{ml})$, BAY11-7082 $(1 \mu \mathrm{M})$ and SP600125 $(5 \mu \mathrm{M})$ for $24 \mathrm{~h}$. The activity of cell proliferation was detected by TransDetect TM Cell Counting Kit (CCK, \# FC101-01, TransGen Biotech).

\section{Cell migration assay}

Cell migration activity was measured by 24-well transwell chambers assay (Transwell, Corning Costar). The cell culture insert with an 8- $\mu \mathrm{m}$ pore size were placed in 24-well plates (lower chamber) containing $500 \mu \mathrm{l}$ of starvation medium with various stimulants, including LPA $(1 \mu \mathrm{M})$, Ki16425 $(1 \mu \mathrm{M})$, EGF $(20$ $\mathrm{ng} / \mathrm{ml})$, SB203580 $(1 \mu \mathrm{M})$, PD98059 $(5 \mu \mathrm{M})$, SP600125 (10 $\mu \mathrm{M})$, BAY11-7082 $(1 \mu \mathrm{M})$, and AG1478 $(1 \mu \mathrm{M})$. Then, cells were placed in the cell culture insert with $200 \mathrm{ul}$ of starvation medium (upper chamber). The 
24-well plate was returned to the cell incubator for $6 \mathrm{~h}$. The cells were pretreated with PTX $(100 \mathrm{ng} / \mathrm{ml})$ for $24 \mathrm{~h}$ and then carried out the cell migration assay.

\section{Animal study}

The male BALB/c nude mice at 4 weeks of age were purchased from Charles River Laboratories (Beijing, China). Tumor xenograft mice model were implemented by stably overexpressing LPA3 in A2780, HO8910 and SKOV3 cells. Each BALB/c nude mouse was injected with $4 \times 10^{6}$ control group cells and experimental group cells at its the right and left flanks, respectively. Each kind of ovarian cancer cells were inoculated into 5-6 mice. The tumor size was measured by vernier caliper weekly until the animals were killed 30 days later. Then the xenograft tumors were subjected to qPCR and immunohistochemistry analyses.

We established a lung metastases model of ovarian cancer in nude mice to evaluate the effects of LPA3 on tumor metastasis. For the intravenous injection, the cancer cells $\left(2 \times 10^{6}\right)$ were injected via the caudal vein to induce lung metastasis. After 30 days, Luciferin (Promega) was injected intraperitoneally into mice at a dose of $150 \mathrm{mg} / \mathrm{kg}$, and within $30 \mathrm{~min}$ after injection, luciferase imaging was performed. Then the mice were killed and counted the number of lung metastatic nodules which were verified by hematoxylin-eosin (HE) staining.

\section{HE staining and immunohistochemistry}

The tumor tissues were dewaxed and stained with an HE staining kit (\#G1120, Solarbio, China). The pathological changes were observed under a microscope. The 4- $\mu \mathrm{m}$ thick sections slides were cut from the paraffin blocks of tumor tissues, then deparaffinized for $15 \mathrm{~min}$ after drying. The slides were incubated with primary antibodies for LPA3 (\#bs-2882R, BIOSS) and Ki-67 (\#bs-23103R, BIOSS) overnight at $4^{\circ} \mathrm{C}$ after antigen retrieval and serum closure. The slides were incubated with secondary antibodies for $60 \mathrm{~min}$. The slides were stained with DAB and HE, then detected by a Zeiss microscope.

\section{The other methods}

The western blot, tissue microarrays and qPCR were performed as described previously $[5,16]$. The cAMP was measured by GloSensor ${ }^{\text {TM }}$ cAMP Assay (Promega). Tissue arrays of human ovarian cancer were purchased from Shanghai Outdo Biotech. The F-actin of ovarian cancer cells were stained with phalloidin-Alexa Fluor 555 (\#C2203S) obtained from Beyotime.

Statistical analysis

All experimental data were performed as mean \pm S.E.M. from at least three independent experiments by GraphPad Prism 7.0 software analysis. Differences with $P<0.05$ were considered statistically significant.

\section{Results}




\section{LPA3 was the only differential expression gene among LPARs in ovarian cancers}

In this study, we first focused on big data by bioinformatics analysis, and then we found that LPA3 was the only differentially expressed LPA receptor among the LPARs in ovarian cancer comparing to the health. According to the volcano map, there were 1236 downregulated genes and 1017 upregulated genes, among which LPA3 expression was upregulated by 12.3 times, but other LPAR family members were not differentially expressed (Fig. 1A). In a statistical analysis of various types of cancers, LPA3 was identified as significantly highly expressed in ovarian cancer (Fig. 1B).

\section{LPA3 highly expressed in ovarian cancer specimens and cell lines}

The bioinformatics studies may not accurately reflect the features of clinical ovarian cancer, we extended the study to detect the expressions of LPA1, LPA2 and LPA3 in patient or normal specimens by immunohistochemistry. LPA1, LPA2 and LPA3 were highly expressed in both normal ovarian tissues and ovarian cancer tissues, but the LPA3 expression was significantly up-regulated in ovarian cancer specimens compared to normal specimens (Fig. 1C and D). Then, we detected the expression of LPARs in several ovarian cancer cell lines. Our data showed that LPA3 was more highly expressed than other LPARs in both A2780 and SKOV3 cells, but LPA1 expression was as high as LPA3 in HO-8910 cells (Fig. 1E and S1).

\section{LPA3 involved in LPA-induced ovarian cancer cell proliferation}

We measured the effects of LPA on the proliferative activity of ovarian cancer cells by CCK assay. Three kinds of ovarian cancer cell lines were stimulated with LPA and EGF. Regardless of the differences in the expression profiles of LPARs in A2780, HO-8910 and SKOV3 cells, LPA increased the cell proliferation activity and Ki16425, as an LPA1/3 antagonist, inhibited cell proliferation, indicating that LPA3 played a key role in LPA-induced cell proliferation (Fig. 2A-B and S2A-D). To further demonstrate this point, we constructed LPA3 overexpression and LPA3 knockdown ovarian cancer cell lines to assess the impact on cell proliferation. The results showed that LPA3 overexpression promoted cell proliferation, while LPA3 knockdown decreased the proliferation of ovarian cancer cells (Fig. 2C-D and S2E-H). Although LPA and EGF, a as positive control, could promote cell proliferation, the combination of LPA and EGF had no synergistic effect (Fig. 2A and D).

\section{LPA3 mediates LPA -induced migration of ovarian cancer cells}

Unlimited self-proliferation and metastasis are the main characteristics of cancer cells. We focused on the cell migration effect of LPA3 in ovarian cancer. Ki16425 remarkably inhibited LPA-induced migration of A2780, SKOV3 and HO-8910 ovarian cancer cells (Fig. 2E and S2I-J). LPA3 knockdown also inhibited cell motility in A2780, SKOV3and HO-8910 ovarian cancer cells (Fig. 2G and S2K-L). LPA3 overexpression significantly increased the cell migration activity compared with that of the control group in A2780, HO8910 and SKOV3 cells (Fig. 2H and S2M-N). We also examined whether LPA3 transactivates epidermal 
growth factor receptor (EGFR) in ovarian cancer cells. The results showed that no synergistic effect was observed between LPA3 and EGFR on ovarian cancer cell migration (Fig. 2F and S2O).

\section{LPA3 exerted in vivo tumorigenesis and metastasis}

The experiments in nude mouse model of human ovarian cancer were used to evaluate the in vivo effect of LPA3 on tumorigenesis and metastasis in ovarian cancer. The overexpression of LPA3 substantially accelerated the tumor growth rate compared to that in the control group, regardless of the ovarian cancer cell type. The mean of tumors volume and mass in the experimental group were remarkably larger than those in the control (Fig. 3A-C and S3). Higher expression of LPA3 and Ki-67 were measured in the LPA3overexpressing cancer cells derived tumor tissues, also indicates LPA3 enhanced the cell proliferation ability (Fig. 3D-F). We assessed the effect of LPA3 on tumor metastasis in vivo, and we further injected A2780-LPA3 overexpressing or control cells into mice via the tail vein. The mice were anesthetized after 30 days, given an intraperitoneal injection of luciferin, and imaged using an in vivo imaging system (IVIS). The lung tissues of nude mice stained with HE showed the morphological characteristics of metastatic tumor. The results further appeared that the fluorescence and the number of pulmonary nodules increased clearly with LPA3 overexpression (Fig. 3G-J).

\section{Signal mechanism for LPA3 mediated actions}

Transactivation of EGFR by LPA3 was not detected in the cell function assay (Fig. 2F and S20), and the measurement of EGFR phosphorylation level also demonstrated that LPA3 did not cause EGFR transactivation (Fig. 4D). In order to clarify the type of G protein coupled by LPA3 in ovarian cancer cells, the cells were stimulated with various inhibitors specific to $G$ proteins. Our findings indicated that LPA via LPA3 induced an inhibition of cAMP accumulation in A2780 cells and PTX, a specific Gi protein inhibitor, blocked LPA-induced cell proliferation and migration (Fig. 4A-C). These results seem to show that LPA stimulated proliferation and migration mediated by LPA3 coupled with Gi-protein in A2780 cells.

Mitogen-activated protein kinase (MAPK) cascades are related to cancer, we examined LPA3-mediated MAPK activation in A2780 cells. The phosphorylation of JNK, p38 MAPK and ERK1/2 were increased in LPA3 overexpression group than those in the control (Fig. 4D). We further used inhibitors specific to MAPK in cell proliferation and migration assay. SB203580 (a p38 inhibitor) markedly suppressed cell migration and proliferation of LPA3-overexpressing cells, and PD98059 (an ERK1/2 inhibitor) significantly attenuated cell migration of the LPA3-overexpressing cells (Fig. 4B-C). However, SP600125 (an JNK inhibitor) hardly affected cell proliferation and migration of the LPA3-overexpressing cells (Fig. 4B-C).

The signaling pathway of Akt and Nuclear factor kappa B (NF-kB) have been linked to ovarian cancer[17, 18]. Importantly, we found that the phosphorylation of Akt and NF-KB were remarkably increased in the LPA3-overexpression group compared with the control (Fig. 4D). While BAY11-7082, a specific inhibitor for NF-KB, abolished LPA3-mediated migration and proliferation of ovarian cancer cells, indicating that NF-KB was involved in LPA3-mediated cell migration and proliferation in ovarian cancer cells (Fig. 4B-C). The F-actin of cellular scaffold was constructed by staining with phalloidin-Alexa Fluor 555 and DAPI 
stained the nuclei blue. Under laser confocal microscope, the F-actin showed red filamentous. LPA3 induced a typical bunched tension filament structure and the number of F-actin was significantly increased to compare with the control, indicating LPA3 signal pathway influenced cell mobility (Fig. 4E).

\section{Discussion}

Ovarian cancer remains a common gynecological malignancy with a high mortality rate worldwide since it was reported by Krukenberg in $1896[12,19,20]$. Since the fact that LPA accumulates in ovarian cancer ascites and increased plasma LPA concentration in ovarian cancer patients had been reported, a lot of research focused on the relationship between the roles of LPA/LPARs and ovarian cancer progression. Park et al reported that LPA via LPA1 and LPA2 induced the migration of ovarian cancer cells [21]. Hadil et al demonstrated that LPA2 and LPA3 were involved in tumor progression through the AKT and ERK signaling pathways, whereas LPA6 was mediated the activation of AKT, affecting cell migration[22]. However, the expression activity of the LPA receptor subtype and its prevalence in ovarian cancer were not clearly demonstrated at the present stage, and which LPA receptor subtypes played a key role in LPAenhanced ovarian cancer progression is still unclear. Therefore, the purpose of our study is to clarify these ambiguities, in order to uncover which LPA receptor subtype is the most important potential therapeutic target for ovarian cancer. In this study, we analyzed both the TCGA and the GTEx database and found that LPA3 was the only differentially expressed gene among LPARs comparing patients with ovarian cancer to healthy controls. The expression of LPA3 increased by 12.3-fold in ovarian cancer samples compared with the control samples, while other LPARs were not. We confirmed that results of LPA1-3 expression in ovarian tumor specimens by immunohistochemistry analysis were consistent with those of the bioinformatics analysis. Our findings suggested that LPA enhanced the cell proliferation and migration response in several ovarian cancer cell lines as well as in LPA3 overexpressing ovarian cancer cell. Conversely, the cell migration and proliferation attenuated by LPA3 knockdown and Ki16425 treatment of ovarian cancer cells. In animal study, LPA3 overexpression markedly increased tumor mass and volume and promoted tumor metastasis in vivo, indicating $L P A 3$ exists a carcinogenicity. Both in vitro and in vivo data suggested that LPA3 plays a key role in LPA-induced actions in ovarian cancer.

One of the challenges in targeting LPARs is the complex array of G-proteins with which they can couple, resulting in multifaceted outcomes. There are different types of $\mathrm{G}$ proteins, including Gq, G13 and Gi couples with LPA3 receptor, depend on the cell types and the environment condition. Excitingly, we found that LPA via LPA3 induced an inhibition of cAMP accumulation in A2780 cells. LPA3 mediated proliferation and migration in A2780 cells were blocked by PTX pretreatment, indicating that LPA3 coupled with Gi protein in the cells.

MAPK, Akt and NF-KB are closely related to the cell proliferation, migration, survival, protein synthesis, glucose metabolism and so on. In the study, we observed that LPA increased the phosphorylation of MAPK, NF-KB and Akt proteins, and LPA-induced effects were strongly inhibited by inhibitors for these proteins, indicating that MAPK, Akt and NF-KB could be involved in LPA3-mediated migration and proliferation in ovarian cancer cells. Activated NF-KB regulates cell proliferation and migration through 
cyclin and metallo matrix proteinase mediated pathways, also regulates cell proliferation survival and migration by stimulating TNF- $a$, IL-6, IL-8, IL-1 and other inflammatory factors secretion and then secondary regulated by these factors in the pro-inflammatory cytokines receptor-mediated manner[23, 24]. Therefore, we hold the opinion that the main way to promote progression of ovarian cancer in response to LPA/LPA3-induced actions are probably by stimulating the expression of pro-inflammatory cytokines.

One hallmark of cancer is the uncontrolled proliferation of cancer cells. Our results indicated that the LPA/LPA3/Gi/p38 or Akt/NF-KB signaling pathway is involved in the proliferation in response to LPA in ovarian cancer cells (Fig. 4F). Metastasis, migration and invasion are essential processes during cancer metastatic progression, which is responsible for approximately $90 \%$ of cancer-associated deaths and is a major problem in cancer treatment[25,26]. We also found that the signaling pathway of LPA/LPA3/Gi/ERK1/2, p38 or Akt/NF-KB was involved in ovarian cancer cells migration (Fig. 4F). Therefore, we propose LPA3 antagonists as potential therapeutic drugs for ovarian cancer.

\section{Conclusion}

In conclusion, based on biological approaches combined with cancer database analysis, we clearly summarized and clarified that the key role of LPA3 in LPA-induced ovarian cancer progression. Our findings suggested that LPA3 antagonists may be a potential therapeutic drug for the ovarian cancer.

\section{Declarations}

\section{Funding}

This work was supported by the National Natural Science Foundation of China (NO. 31860253囚 31971219).

\section{Conflicts of interest}

The authors report no conflicts of interest for this work.

\section{Availability of data and material}

All data included in this study are available upon request by contact with the corresponding author.

Code availability (software application or custom code)

Not applicable.

\section{Authors' contributions}

Alatangaole Damirin and Pengfei Zhao contributed to design the study. Pengfei Zhao, Qingru Yun, Aodungerile Li, Rong Li and Yali Yan performed the experiment and collected data. Yuewu Wang 
performed the animal experiments. Pengfei Zhao and Hongju Sun analyzed the data and wrote the manuscript. Alatangaole Damirin revised manuscript critically for important intellectual content and final approval of the version submitted for publication. All the authors approved the final manuscript.

\section{Ethics approval}

All experiments used in this study were performed with ethical standards that the Declaration of Helsinki and national and international guidelines. This investigation was reviewed and approved by the Institutional Animal Care and Use Committee of Inner Mongolia University (approval number 2020001).

Consent to participate (include appropriate statements)

Not applicable.

Consent for publication (include appropriate statements)

Not applicable.

\section{Acknowledgments}

We are grateful to Prof. Fumikazu Okajima at Gunma University for a generous gift of the Ki16425.

\section{References}

1. Xu Y. Targeting Lysophosphatidic Acid in Cancer: The Issues in Moving from Bench to Bedside. Cancers. 2019; 11.

2. Tang X, Brindley DN. Lipid Phosphate Phosphatases and Cancer. Biomolecules. 2020; 10.

3. Ha JH, Radhakrishnan R, Jayaraman M, et al. LPA Induces Metabolic Reprogramming in Ovarian Cancer via a Pseudohypoxic Response. Cancer research. 2018;78:1923-34.

4. Wu D, Wu F, Li B, et al. EZH2 promotes the expression of LPA1 by mediating microRNA-139 promoter methylation to accelerate the development of ovarian cancer. Cancer cell international. 2020;20:551.

5. Zhao PF, Wu S, Li Y, et al. LPA receptor1 antagonists as anticancer agents suppress human lung tumours. Eur J Pharmacol. 2020;868:172886.

6. Shi W, Zhang C, Ning Z, et al. CMTM8 as an LPA1-associated partner mediates lysophosphatidic acid-induced pancreatic cancer metastasis. Annals of translational medicine. 2021;9:42.

7. Ueda N, Minami K, Ishimoto K, et al. Effects of lysophosphatidic acid (LPA) receptor-2 (LPA2) and LPA3 on the regulation of chemoresistance to anticancer drug in lung cancer cells. Cellular signalling. 2020;69:109551.

8. Minami K, Ueda N, Ishimoto K, et al. Cooperation of G12/13 and Gi proteins via lysophosphatidic acid receptor-2 (LPA2) signaling enhances cancer cell survival to cisplatin. Biochem Biophys Res Commun. 2020;532:427-32. 
9. Xu Y, Shen Z, Wiper DW, et al. Lysophosphatidic acid as a potential biomarker for ovarian and other gynecologic cancers. Jama. 1998;280:719-23.

10. Bai CQ, Yao YW, Liu CH, et al. Diagnostic and prognostic significance of lysophosphatidic acid in malignant pleural effusions. Journal of thoracic disease. 2014;6:483-90.

11. Zeng R, Li B, Huang J, et al. Lysophosphatidic Acid is a Biomarker for Peritoneal Carcinomatosis of Gastric Cancer and Correlates with Poor Prognosis. Genetic testing molecular biomarkers. 2017;21:641-8.

12. Siegel RL, Miller KD, Jemal A. Cancer statistics. 2020. CA: a cancer journal for clinicians. 2020; 70: 7-30.

13. Ray U, Chowdhury SR, Vasudevan M, et al. Gene regulatory networking reveals the molecular cue to lysophosphatidic acid-induced metabolic adaptations in ovarian cancer cells. Molecular oncology. 2017; 11.

14. Park J, Jang JH, Oh S, et al. LPA-induced migration of ovarian cancer cells requires activation of ERM proteins via LPA1 and LPA2. Cellular signalling. 2018; 44.

15. Li GC, Qin XL, Song HH, et al. Upregulated microRNA-15b alleviates ovarian cancer through inhitbition of the PI3K/Akt pathway by targeting LPAR3. J Cell Physiol. 2019;234:22331-42.

16. Azim HA Jr, Peccatori FA, Brohee S, et al. RANK-ligand (RANKL) expression in young breast cancer patients and during pregnancy. Breast cancer research: BCR. 2015;17:24.

17. Ediriweera MK, Tennekoon KH, Samarakoon SR. Role of the PI3K/AKT/mTOR signaling pathway in ovarian cancer: Biological and therapeutic significance. Semin Cancer Biol. 2019;59:147-60.

18. Kan Y, Liu J, Li F. High Expression of Nuclear Transcription Factor-kappaB is Associated with Cisplatin Resistance and Prognosis for Ovarian Cancer. Cancer management research. 2020;12:8241-52.

19. Haakonsen $M$, Gudmundsen TE, Lilleng R [Krukenberg tumor. A seldom considered condition?]. Tidsskrift for den Norske laegeforening: tidsskrift for praktisk medicin, ny raekke. 1997; 117: 2180-1.

20. Kyo S, Ishikawa N, Nakamura K, et al. The fallopian tube as origin of ovarian cancer: Change of diagnostic and preventive strategies. Cancer medicine. 2020;9:421-31.

21. Park J, Jang JH, Oh S, et al. LPA-induced migration of ovarian cancer cells requires activation of ERM proteins via LPA1 and LPA2. Cellular signalling. 2018;44:138-47.

22. Onallah H, Davidson B, Reich R. Diverse Effects of Lysophosphatidic Acid Receptors on Ovarian Cancer Signaling Pathways. Journal of oncology. 2019; 2019: 7547469.

23. Soleimani A, Rahmani F, Ferns GA, et al. Role of the NF-KB signaling pathway in the pathogenesis of colorectal cancer. Gene. 2019;726:144132.

24. Wang L, Niu Z, Wang X, et al. PHD2 exerts anti-cancer and anti-inflammatory effects in colon cancer xenografts mice via attenuating NF-KB activity. Life sciences. 2019;242:117167.

25. Chi HC, Tsai CY, Tsai MM, et al. Roles of Long Noncoding RNAs in Recurrence and Metastasis of Radiotherapy-Resistant Cancer Stem Cells. International journal of molecular sciences. 2017; 18. 
26. Wu D, Yang $X$, Peng $H$, et al. OCIAD2 suppressed tumor growth and invasion via AKT pathway in Hepatocelluar carcinoma. Carcinogenesis. 2017;38:910-9.

\section{Figures}

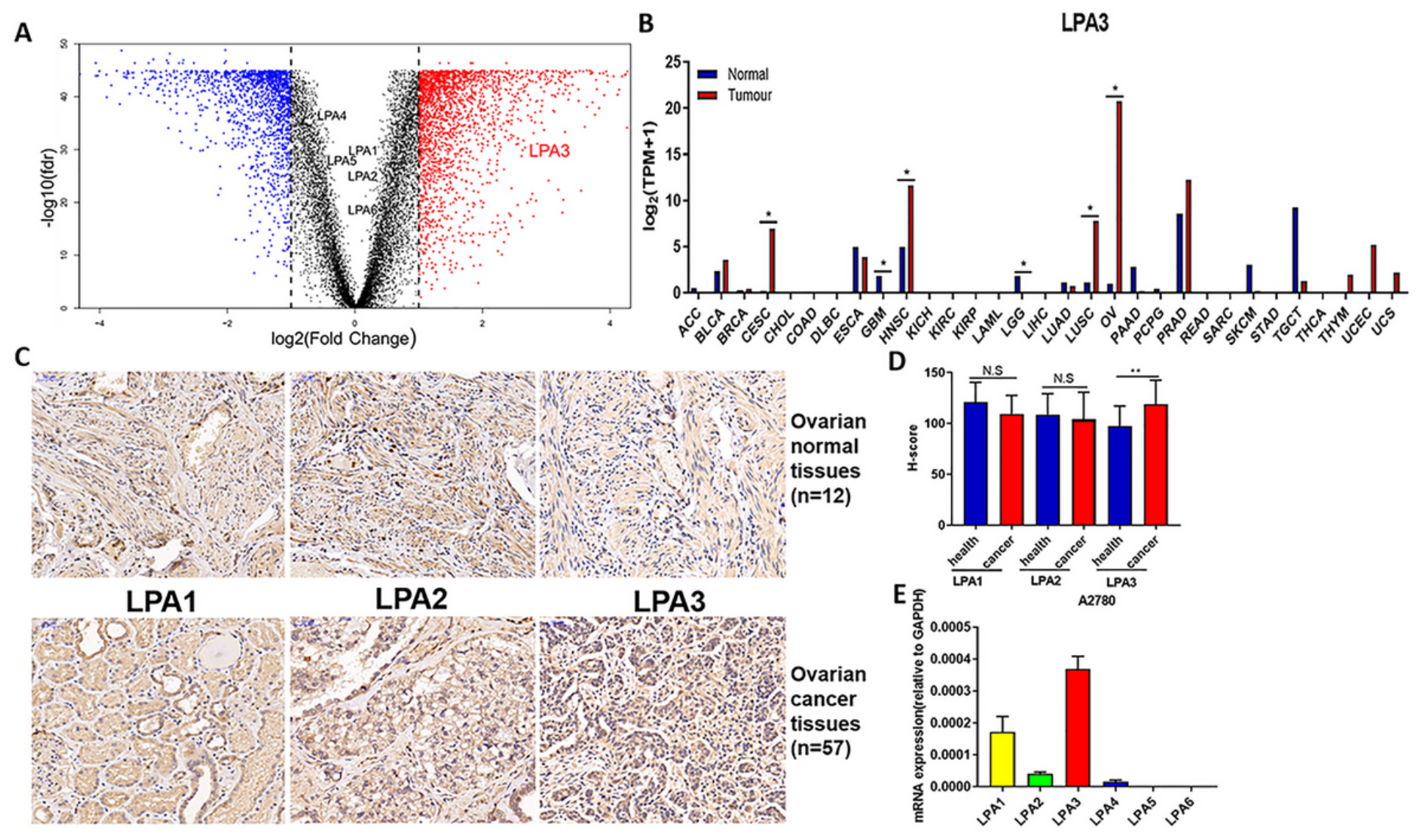

Figure 1

LPA3 highly expressed in ovarian cancer tissues and cells. (A) Volcano map of differentially expressed genes in ovarian cancer samples vs control samples. (B) The expression of LPA3 in a variety of types of cancer. (C) Comparison of LPA1, LPA2 and LPA3 expression in ovarian tissues $(n=57)$ and normal tissues $(n=12)$ using immunohistochemistry. (D) The expression of LPA1, LPA2 and LPA3 as determined by immunohistochemistry using the $\mathrm{H}$-scores of ovarian cancer patient tissues and healthy tissues. $(E)$ LPARs expression profiles were detected in ovarian cancer cell lines by qPCR. 
A
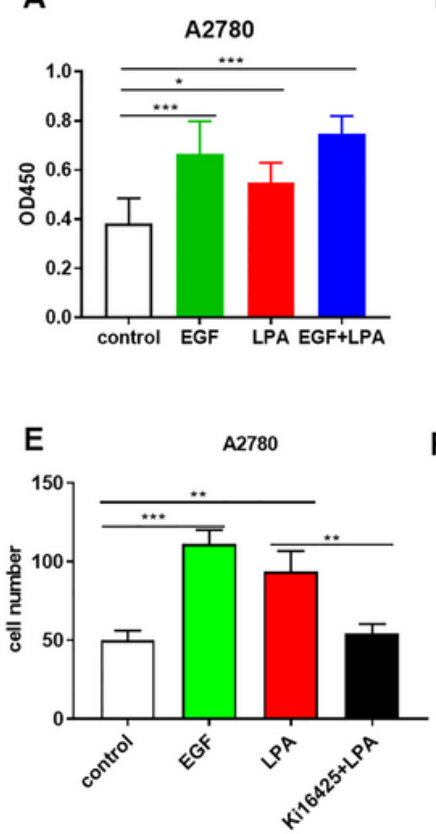

B

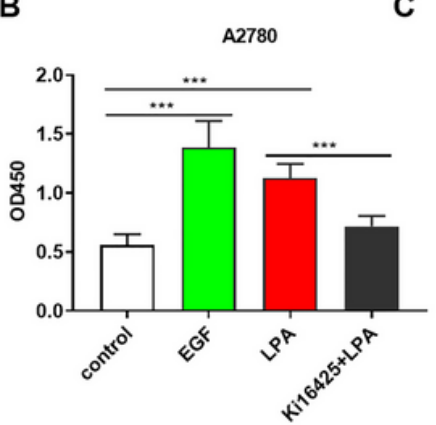

F

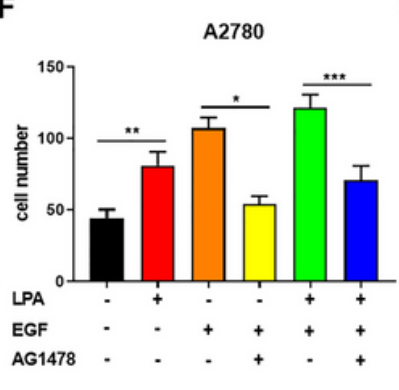

c

G

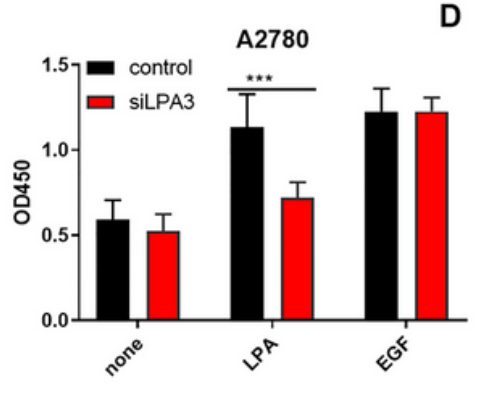

A2780

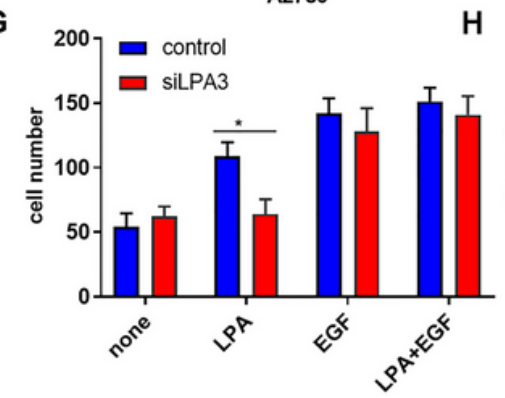

D

H
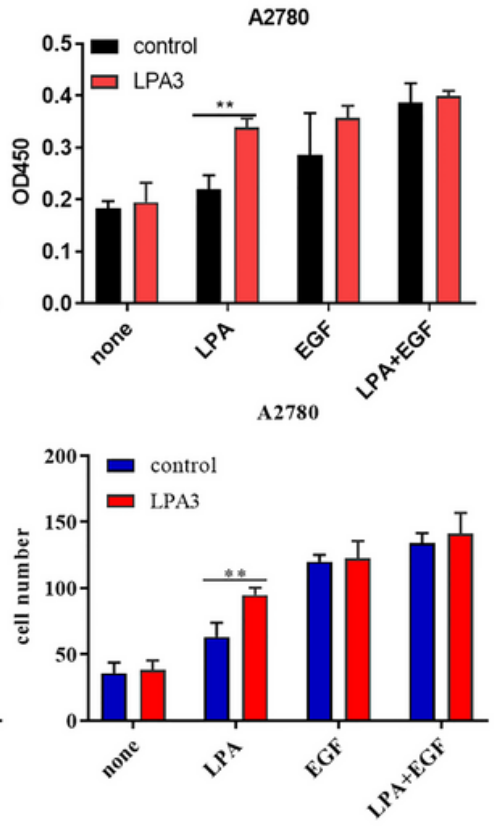

Figure 2

LPA3 mediated proliferation and migration in response to LPA in ovarian cancer cell. (A-B) A2780 cells were treated with $1 \mu \mathrm{M} \mathrm{LPA}, 1 \mu \mathrm{M} \mathrm{Ki} 16425$ and $20 \mathrm{ng} / \mathrm{ml}$ EGF for $24 \mathrm{~h}$. Then, cell viability was measured by CCK assay. (C-D) LPA3-overexpression and LPA3 knockdown ovarian cancer cells were stimulated with $1 \mu \mathrm{M}$ LPA and $20 \mathrm{ng} / \mathrm{ml}$ EGF for $24 \mathrm{~h}$, and cell proliferation was detected by CCK assay. (E-F) A2780 cells were incubated with $1 \mu \mathrm{M}$ Ki16425, $1 \mu \mathrm{M} \mathrm{AG} 1478,1 \mu \mathrm{M} \mathrm{LPA}$, and $20 \mathrm{ng} / \mathrm{ml}$ EGF for $6 \mathrm{~h}$ to measure cell migration by Transwell assay. (G-H) LPA3-overexpressing and LPA3-knockdown ovarian cancer cells were treated with $1 \mu \mathrm{M}$ LPA and $20 \mathrm{ng} / \mathrm{ml}$ EGF for $6 \mathrm{~h}$. Then, cell motility was assessed by the Transwell assay. 
A A2780
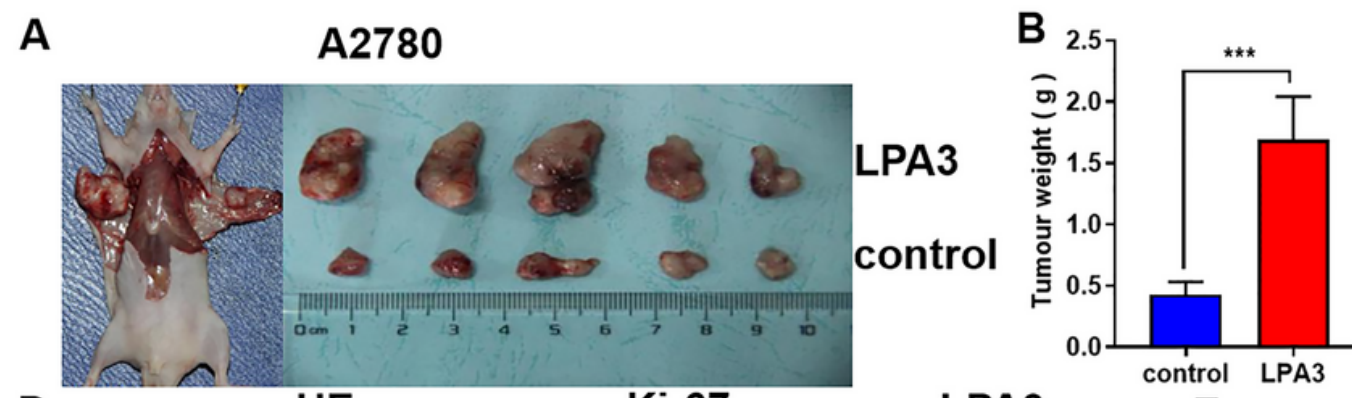

C

D

HE

Ki-67

LPA3

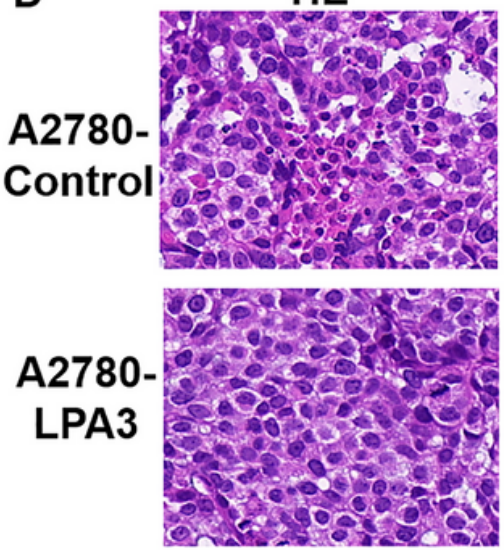

G

A2780-control

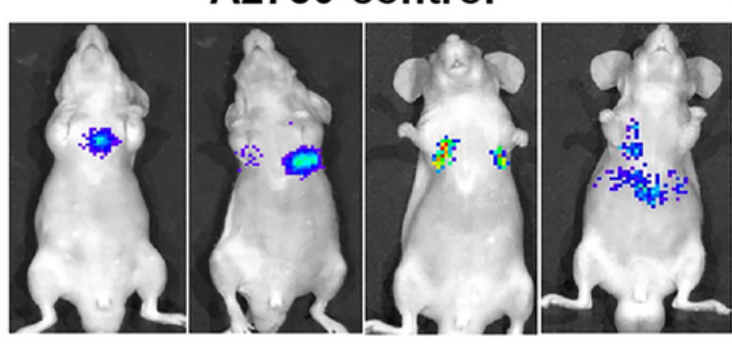

A2780-LPA3

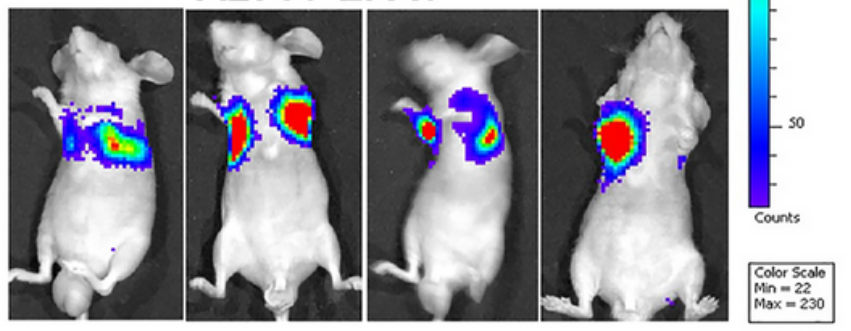

$\mathrm{H}$

E

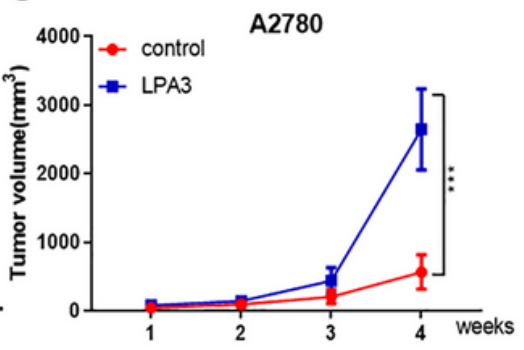

F

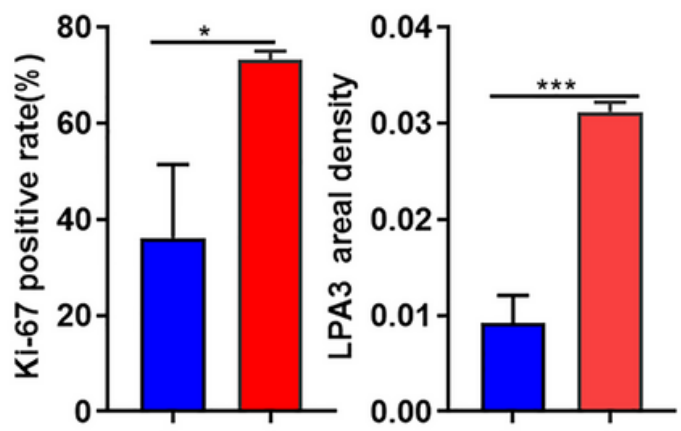

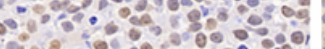

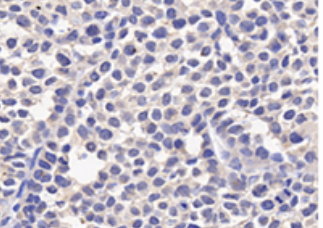

301090908
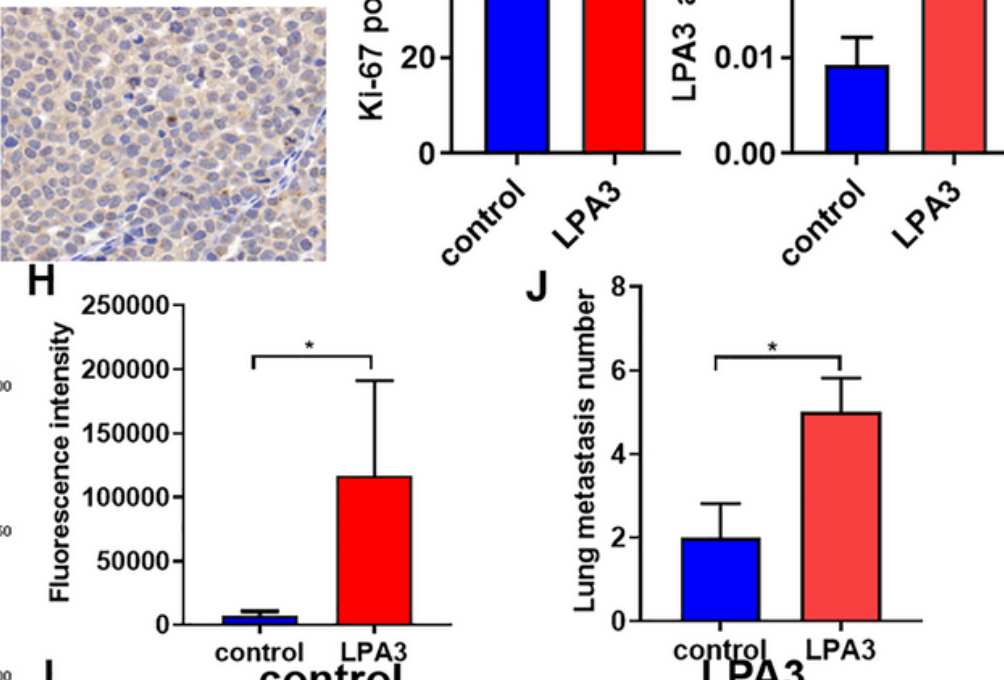

control
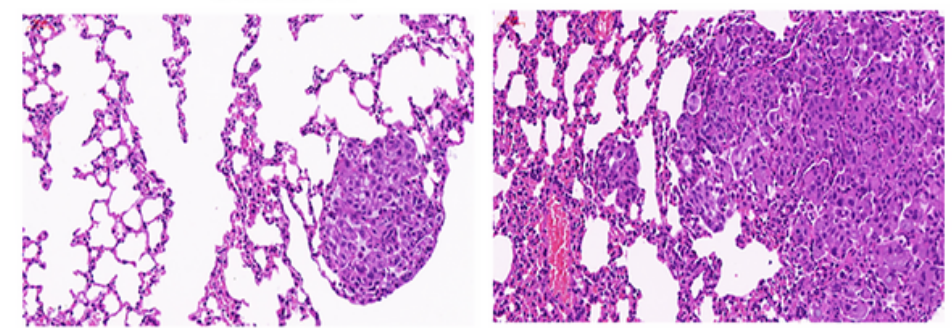

Figure 3

LPA stimulates tumor growth and metastasis via LPA3 in vivo. (A) Image of tumors in the xenografted mice model: LPA3-overexpressing ovarian cancer cells and controls were injected subcutaneously into the left and right flanks of mice ( $n=5)$. (B) The tumors were dissected and weighed at on day 30. (C) The tumor volume was monitored and measured once a week. (D) Histological morphology analysis of tumor tissue sections from A2780 tumor-bearing mice stained with HE. Immunostaining of tumor tissue sections from A2780 tumor-bearing mice and antibodies against LPA3 and Ki-67 (400x). (E-F) Imagebased quantification of LPA3 and Ki-67. (G) Images of nude mice with a lung metastasis $(n=4)$. $(H)$ 
Quantification of fluorescence intensity. (I) The photos of HE-stained lung sections. (J) Statistical analysis of lung metastatic nodules.

A
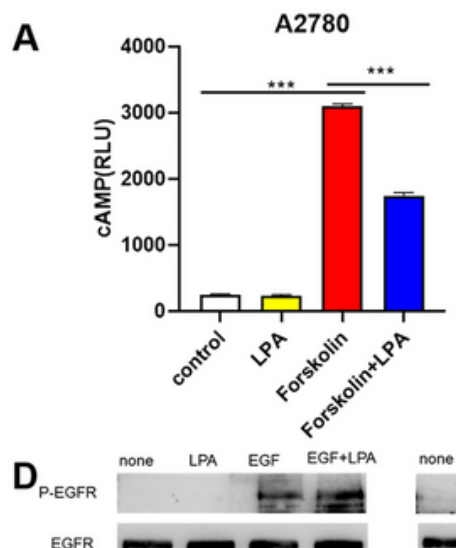

EGFR

P.ERK $1 / 2$

ERK $1 / 2$

P.JNK

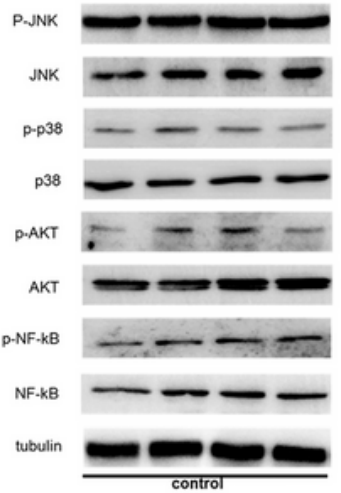

F
B 듬 ${ }_{\mathrm{LAB}}^{\text {contol }}$

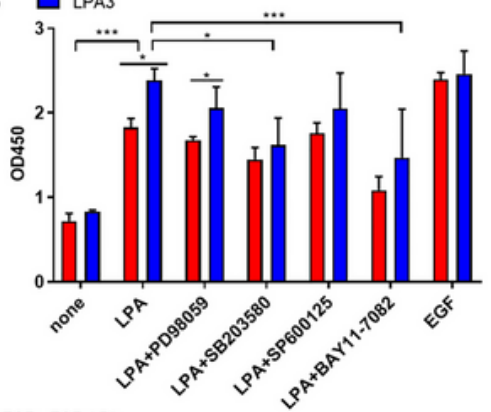

E
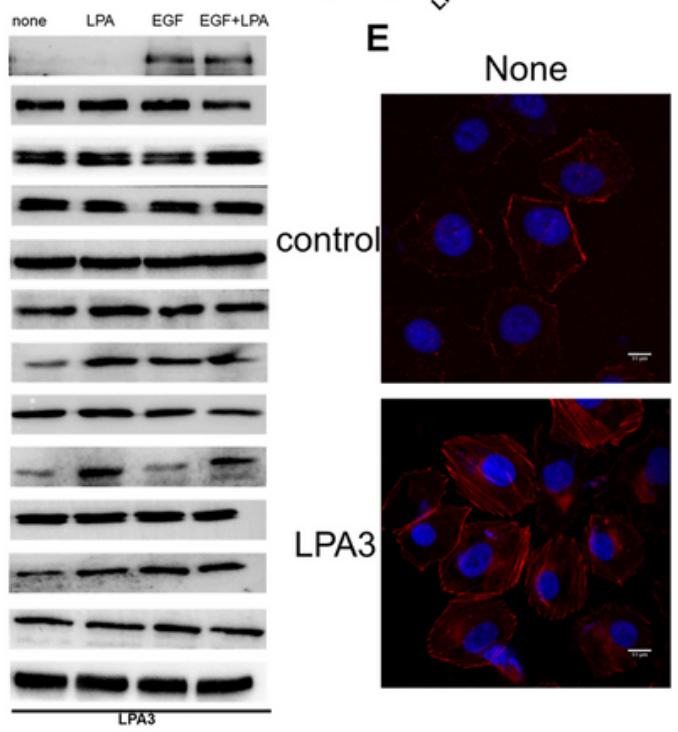

C 믐 ${ }_{\mathrm{LPA}}^{\mathrm{contr}}$

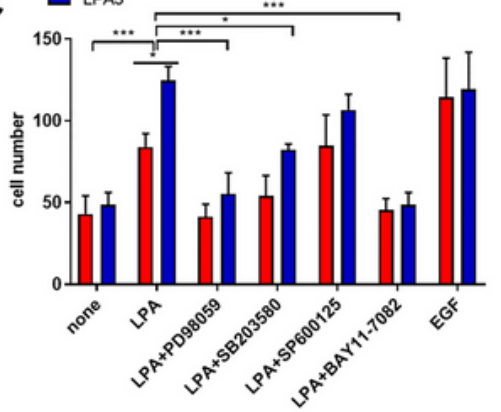

LPA

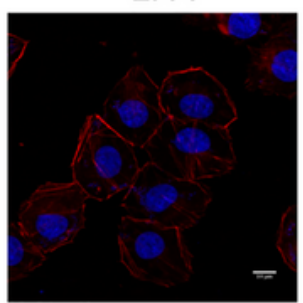

EGF
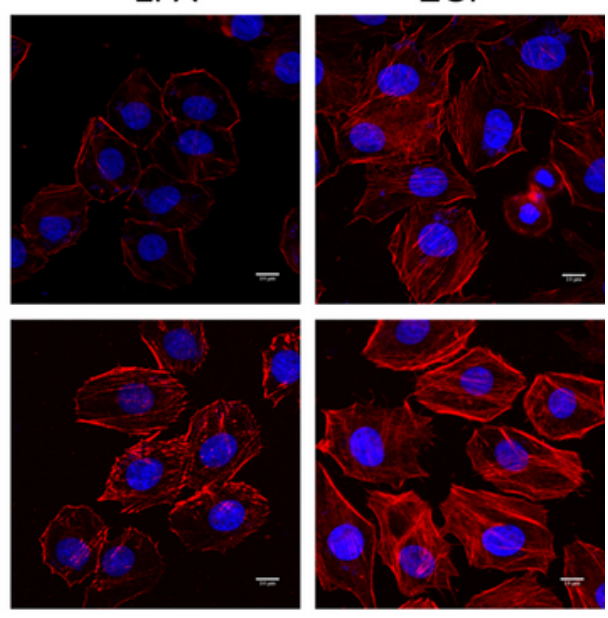

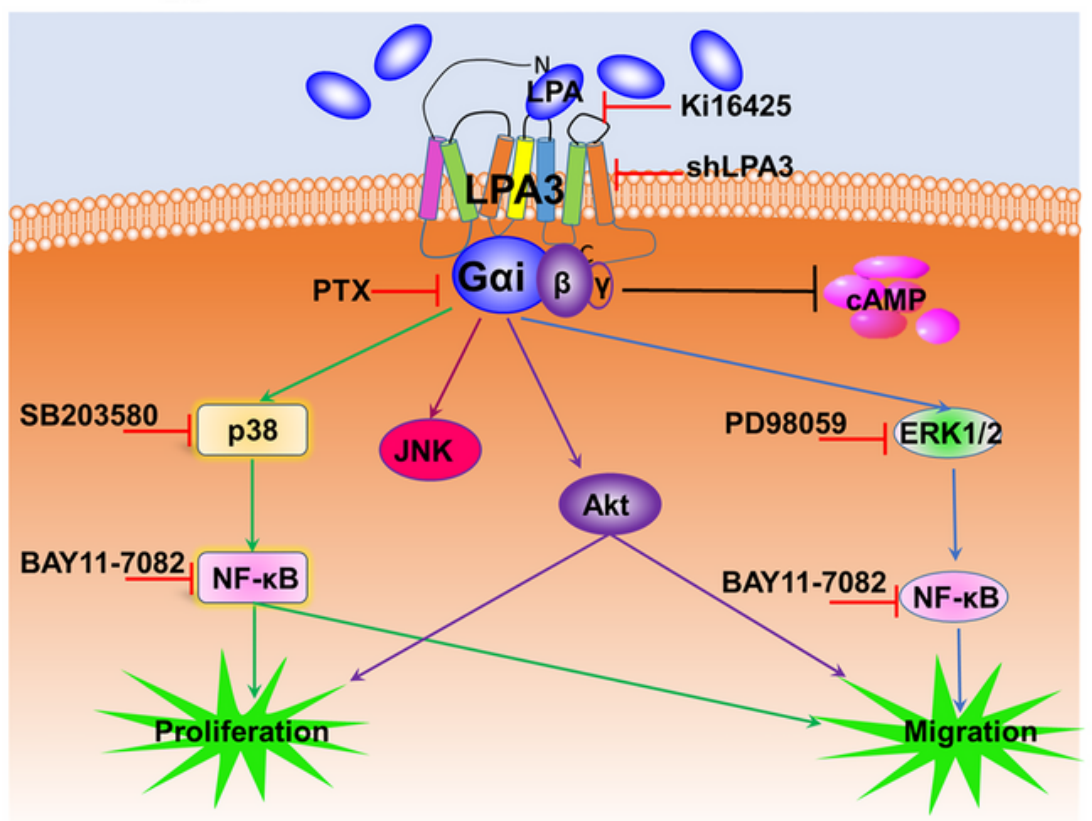

Figure 4

Signaling pathways involved in A2780 cell proliferation and migration induced by LPA. (A)The LPA3overexpressing A2780 cells were stimulated with or without $1 \mu \mathrm{M}$ LPA and $1 \mu \mathrm{M}$ Forskolin. Then, read luminescence by Multifunctional microplate reader. (B) LPA3-overexpression A2780 cells and control 
were stimulated with $1 \mu \mathrm{M}$ LPA, $5 \mu \mathrm{M}$ PD98059, $1 \mu \mathrm{M}$ SB203580, $5 \mu \mathrm{M}$ SP600125, $1 \mu \mathrm{M}$ BAY11-7082, 100 $\mathrm{ng} / \mathrm{ml}$ PTX and $20 \mathrm{ng} / \mathrm{mL}$ EGF for $24 \mathrm{~h}$. The cell proliferation was detected by the CCK assay. (C) The control and LPA3-overexpressing cells were treated with $1 \mu \mathrm{M}$ LPA or $20 \mathrm{ng} / \mathrm{ml}$ EGF for $6 \mathrm{~h}$ after pretreatment with or without different inhibitors, namely, PTX, PD98059, SB203528, SP600125, and BAY11-7082 for the indicated times. Then, using the Transwell assay, the migration of ovarian cancer cells was assessed. (D) LPA3-overexpressing A2780 cells and the control were stimulated with $1 \mu \mathrm{M}$ LPA and $20 \mathrm{ng} / \mathrm{ml}$ EGF for $15 \mathrm{~min}$. The effects of LPA on MAPKs, AKT, and NF-KB phosphorylation were measured by western blotting. (E) Effect of LPA3 on the cytoskeleton in A2780 cells ( $\times 100)$. (F) LPA effects mediated by the LPA3 signaling pathway in ovarian cancer cells.

\section{Supplementary Files}

This is a list of supplementary files associated with this preprint. Click to download.

- FigS1.tif

- FigS2.tif

- FigS3.tif 